\title{
Experimental and finite element analysis of vibrations of a rotating annular plates
}

\author{
M. Tufekci ${ }^{1^{*}}$, O. E. Genel ${ }^{2}$, O. Oldac ${ }^{3}$ \\ 1, 2, 3 Istanbul Technical University, Istanbul, Turkey
}

\author{
Index Terms \\ Rotating Disk \\ Annular Plate \\ Transverse Vibrations \\ Finite Element \\ Experimental
}

Received: 12 July 2017

Accepted: 8 August 2017

Published: 4 December 2017

\begin{abstract}
The aim of this study is to investigate the small amplitude transverse vibration characteristics of an annular thin plate (disk) which rotates about its axis with the rotational speed. The disk is clamped at the inner circumference and free at the outer circumference. This study contains two sections, first one is the experimental investigation of the transverse dynamical behavior of the disk at various rotational speeds, the second section includes modelling the disk numerically using Finite Element Method (FEM). For the experimental part of the study, a Hard Disk Drive (HDD) is used. The disk is rotated with various rotational speeds and measurements are made using non-contacting systems for each rotational speed value. For the numerical part, a finite element model is built and the natural frequencies with the related mode shapes are obtained. The natural frequencies are plotted for various rotational speeds. Using the data obtained from the results of the study Campbell diagrams are drawn. In order to validate the results of this study, the numerical and experimental results are compared to each other and also to the literature.
\end{abstract}

(C) 2017 The Author(s). Published by TAF Publishing.

\section{INTRODUCTION}

Rotating disks have extensive application areas for many years. Turbines, flywheels, grinding wheels, automotive brake disks, wood saw cutting machines and computer data storage devices can be given as examples. Due to rotating at high speeds and having unavoidable manufacturing imperfections, vibration problems in these structures must be taken into consideration. In forestry sector, vibration of electric wood saw cutting machine can cause significant material losses or in hard disks, transverse vibrations of rotating disk can affect the precision of read/write head's activities - disk contact and that contact can cause excessive loads on disk. A hard disk drive is expected to be precise and fast. This requires higher rotation speeds for performing fast. So predictions with better accuracy on its dynamic behavior are crucial for higher precision.

Earlier theoretical study about rotating disk problem is presented by Lamb and Southwell [1]. Their study is based on linear theory while neglecting bending stiffness.
Southwell [2] investigated natural frequencies of a rotating disk which has clamped-free boundary conditions at inner and outer edges, respectively. Besides linear theory, Nowinski [3] presented nonlinear transverse vibrations of rotating disks on the basis of von Karman plate theory. Mignolet et al. [4] used perturbation techniques to predict free vibrational properties of rotating disks with and without hole at its center. Nayfeh et al. [5] carried out a research on transverse vibrations of rotating disks using the method of multiple scales and solved the problem for both linear and nonlinear vibrations.

In literature, there are a few experimental researches on rotating disks. Bashmal et al. [6] used RayleighRitz method to predict in-plane and out-of-plane vibrational characteristics of annular circular plates with various boundary conditions and supported their predictions with laboratory experiments. Kajiwara and Hosoya [7] performed an experiment with a contactless excitation system which is based on laser ablation technique and measured

\footnotetext{
* Corresponding author: M. Tufekci

${ }^{\dagger}$ Email: tufekcime@itu.edu.tr
} 
the vibrations with Laser Doppler Vibrometer (LDV). Khorasany and Hutton [8] investigated vibrations of rotating thin disks with and without space fixed lateral loaded conditions.

There have been some studies that investigated behavior of such circular plates using numerical methods. Cole and Benson [9] developed a numerical approach to determine the most dominant modes of the spinning disks. The proposed method converged faster to the real response than other commonly used methods. Kirkhope and Wilson [10] applied finite element method approach to rotating thin disk problem by taking advantage of axisymmetric properties and using annular elements. In addition to the vibration characterization, stress analysis is also performed in the study. Xu and Guo [11] developed a model with larger scale and modeled hard disk assembly using finite element method. They investigated the effect of head-actuator assembly and the elastic bearing on dynamic characteristics of hard disk drive. Also, to support their research, they performed a modal testing experiment on head-actuator assembly. One crucial point in mechanical analysis of rotating disk is the investigation of travelling waves. Kim and Lee [12] built a modal control scheme for rotating disks using finite dimensional sub-system model considering some dominant and significant travelling waves and analyzed the stability and spillover effect.

In this study, transverse vibrations of a hard disk are investigated both numerically and experimentally. Natural frequencies and critical speeds of hard disk are calculated at different speeds using FEM. Also, for validating results in terms of consistency, an experiment is performed. Finally, both numerical and experimental results are com- pared with analytical results. For comparison, an analytical approach which is presented by Oldac et al. [13] is used.

\section{EXPERIMENTAL STUDY}

Main objective of experiment is obtaining autospectrums of vibration signals that are collected from a hard disk at various speeds. Hard disk used in the experiments is Seagate Barracuda ST2000DM001 model. The thickness of the disk is $1.3 \mathrm{~mm}$ and its inner and outer diameters are 32.5 $\mathrm{mm}$ and $95 \mathrm{~mm}$, respectively. After eliminating the sheet metal cap, it is clamped to the fixture. Most of the modern hard disks are operated at $7200 \mathrm{rpm}$. In this experiment, operating speed is increased from 0 to $8000 \mathrm{rpm}$ with 2000 rpm increments.

In terms of modularity and simplicity, experimental setup is divided into three subsystems. The first one is speed control system. In order to be able to operate the hard disk at various speeds, a servo tester and Electronic Speed Controller (ESC) are used. The second subsystem is speed feedback system. For setting up a reliable measurement system, speed feedback information system is designed. Brüel \& Kjaer 4507 B type accelerometer is located on top of the hard disk casing and the existing imperfections of the disk rotation frequency are measured by accelerometer and tracked in PULSE environment. With the help of this system, user makes sure that whether the system is rotating at the desired speed. Final subsystem is response measurement system. For collecting the vibration data, Polytec CLV-2534 Compact Laser Vibrometer is used. Collected signal is analyzed in Brüel \& Kjaer 3050-A-060 LAN XI analyzer and visualized in PULSE Labshop. Then, data sets are saved as UFF file and plotted in MATLAB. Entire measurement system is represented both conceptually and physically in Figure 1.

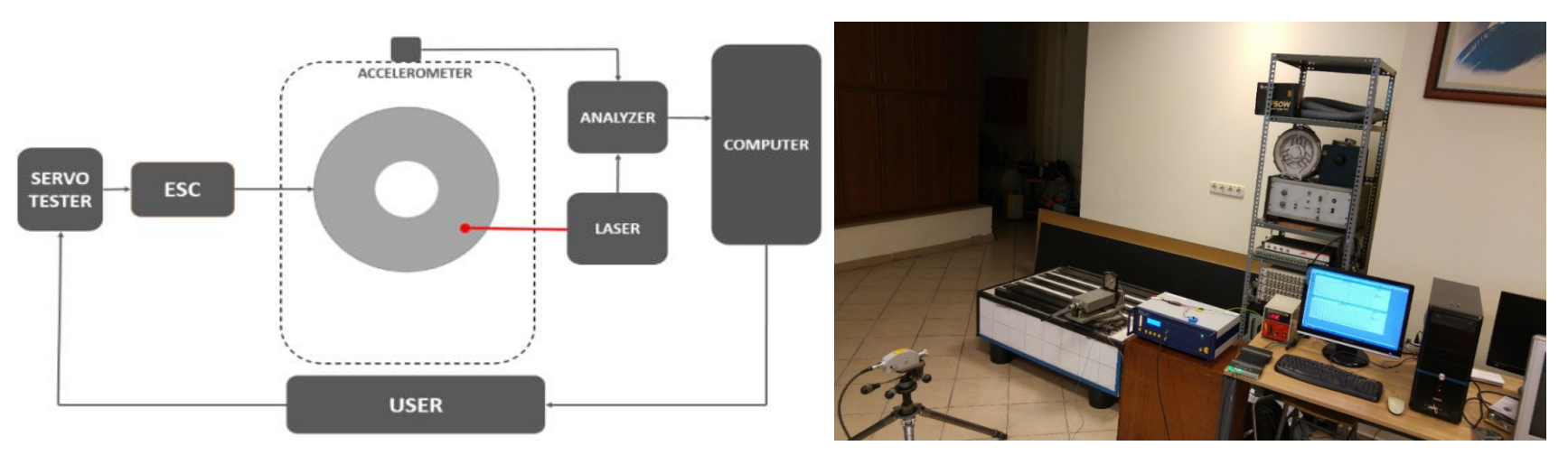

Fig. 1. Conceptual and physical representation of measurement system 
First, the natural frequencies of the stationary hard disk are determined, Figure 2. In rotating case, measurements are taken from 0 to $8000 \mathrm{rpm}$ with $2000 \mathrm{rpm}$ incre- ments. For mode 1 and mode 4 , obtained measurements are represented in Figure 3 and Figure 4, respectively.

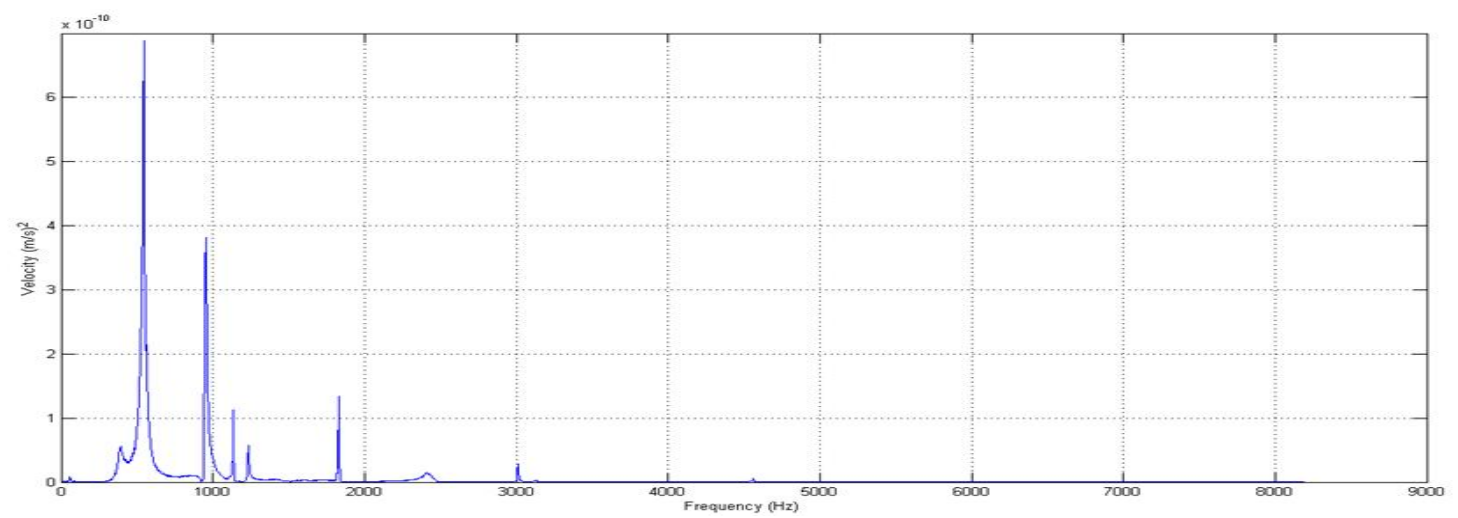

Fig. 2 . Natural frequencies of stationary disk
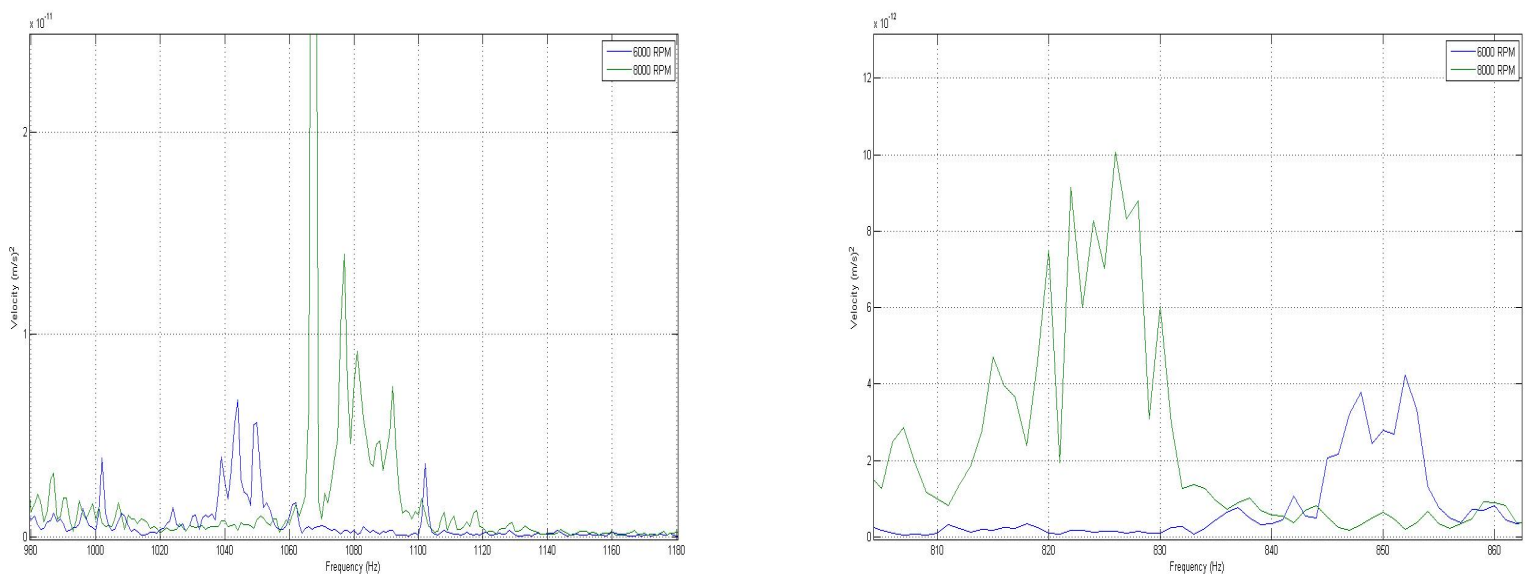

Fig. 3 . Forward and backward travelling wave of mode 1 at 6000 and $8000 \mathrm{rpm}$
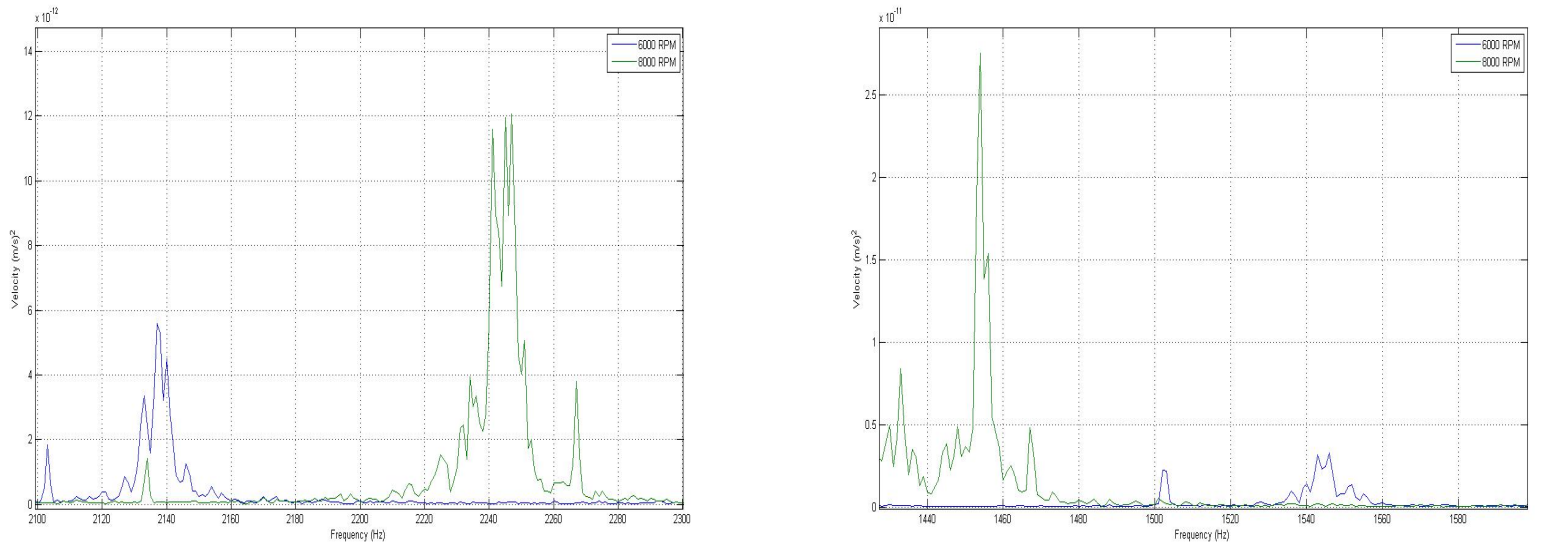

Fig. 4 . Forward and backward travelling wave of mode 4 at 6000 and $8000 \mathrm{rpm}$ 
Natural frequencies which consist of Forward Travelling Waves (FTW) and Backward Travelling Waves (BTW) for various speeds are represented in Table 1. In rotating case, natural frequencies of mode 2 are the same for FTW and BTW at each rpm. The reason for this is mode 2 cor- responds to $(0,0)$ mode shape. This means that it has no nodal diameters and nodal circles. Therefore, instead of observing two different natural frequency values (FTW and BTW), only one natural frequency value is observered for each speed.

TABLE 1

EXPERIMENTAL RESULTS FOR VARIOUS SPEEDS

\begin{tabular}{|c|c|c|c|c|c|c|c|}
\hline Rotation Speed (RPM) & Wave Type & $\begin{array}{c}\text { Mode } 1(\mathrm{~Hz}) \\
(0,1)\end{array}$ & $\begin{array}{c}\text { Mode } 2(\mathrm{~Hz}) \\
(0,0)\end{array}$ & $\begin{array}{c}\text { Mode } 3(\mathrm{~Hz}) \\
(0,2)\end{array}$ & $\begin{array}{c}\text { Mode } 4(\mathrm{~Hz}) \\
(0,3)\end{array}$ & $\begin{array}{c}\text { Mode } 5(\mathrm{~Hz}) \\
(0,4)\end{array}$ & $\begin{array}{c}\text { Mode } 6(\mathrm{~Hz}) \\
(0,5)\end{array}$ \\
\hline 0 & - & 955 & 1136 & 1232 & 1830 & 3009 & 4560 \\
\hline 2000 & BTW & 906 & 1141 & 1174 & 1722 & 2870 & 4387 \\
\hline \multirow[t]{2}{*}{4000} & FTW & 1009 & 1148 & 1350 & 2032 & 3276 & 4913 \\
\hline & BTW & 880 & 1148 & 1101 & 1641 & 2741 & 4227 \\
\hline 6000 & BTW & 852 & 1153 & 1044 & 1544 & 2624 & 4068 \\
\hline \multirow[t]{2}{*}{8000} & FTW & 1077 & 1160 & 1484 & 2245 & 3553 & 5218 \\
\hline & BTW & 820 & 1160 & 971 & 1454 & 2500 & 3907 \\
\hline
\end{tabular}

\section{FINITE ELEMENT ANALYSIS}

Numerical methods provide fast and acceptably accurate solutions for many complex cases, independent of being analytically solvable. FEM is one of the most powerful and one of the most widely used numerical methods. There are various commercial software packages in the market to make finite element calculations user freindly, fast and practical. Abaqus lies in the better part of the spectrum of the softwares offering more functions, capabilities and flex- ibility. Therefore Abaqus is used for this study to build the desired finite element model.

In this study it is aimed to build a finite element model that is reliable without requiring much computational resources or time. The disk is modelled as shell using shell type of quadratic elements. Therefore reduced integration is enabled to make the calculation faster. These elements consist of 8 nodes each and there are 6 degrees of freedom per node. In order to be able to calculate higher modes of vibration, 16 elements are used in the radial direction. Figure 5 shows the meshed disk.
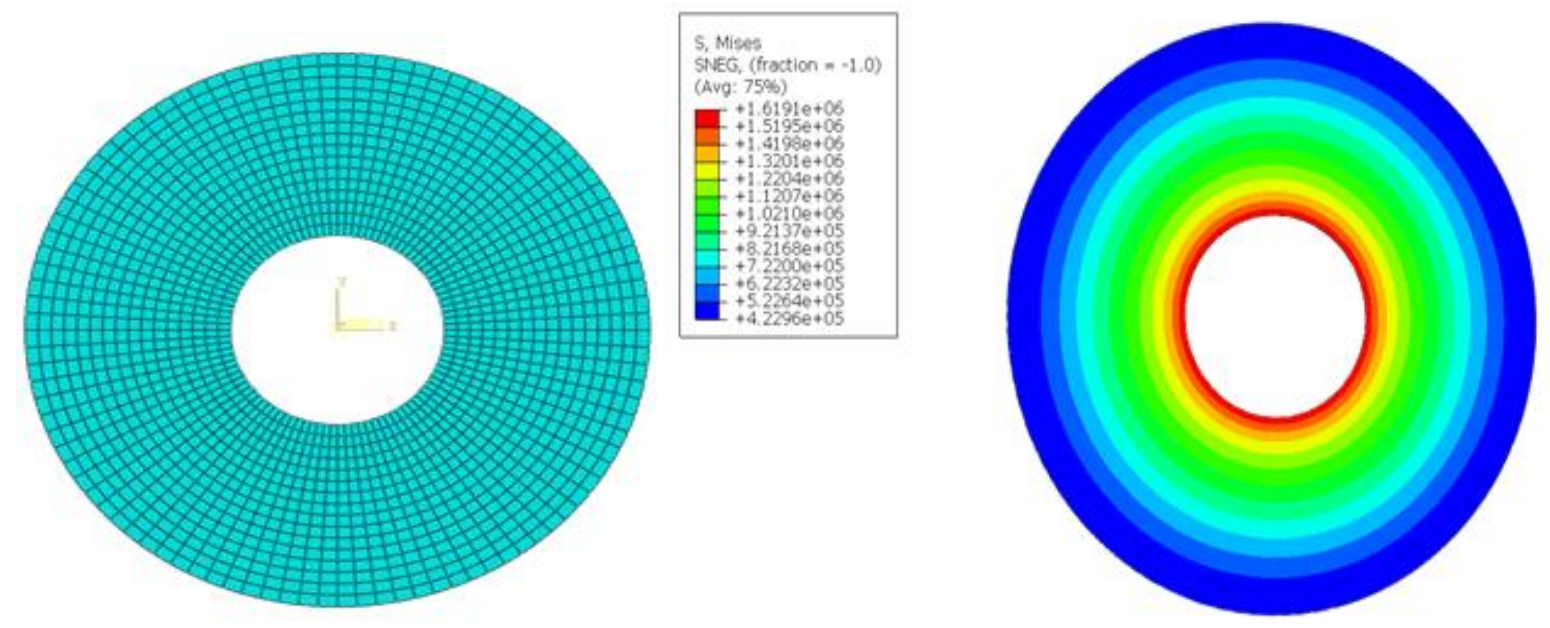

Fig. 5 . Meshed disk with S8R element type and static step stress results 
The hard disk geometry and material properties given in the experimental study are used in the analysis. But various disk examples with various rotational speeds are analyzed with the built model.

For modeling the hard disk, the inner edge of the disk is fixed as encastre and centrifugal body forces are applied depending on the rotational speed to simulate rotation.

There are 2 steps following each other. The first one is a general static step with nonlinear geometry option enabled to include the effects of loads on the related stiffness matrices. Figure 5 shows an example stress distribution on the disk when rotating with its regular operating rotational speed of $7200 \mathrm{rpm}$. The second step is the frequency calculation step. The frequency step calculates the natural frequencies of updated stiffness matrix that comes out as a result of the static step. So the frequencies calculated are
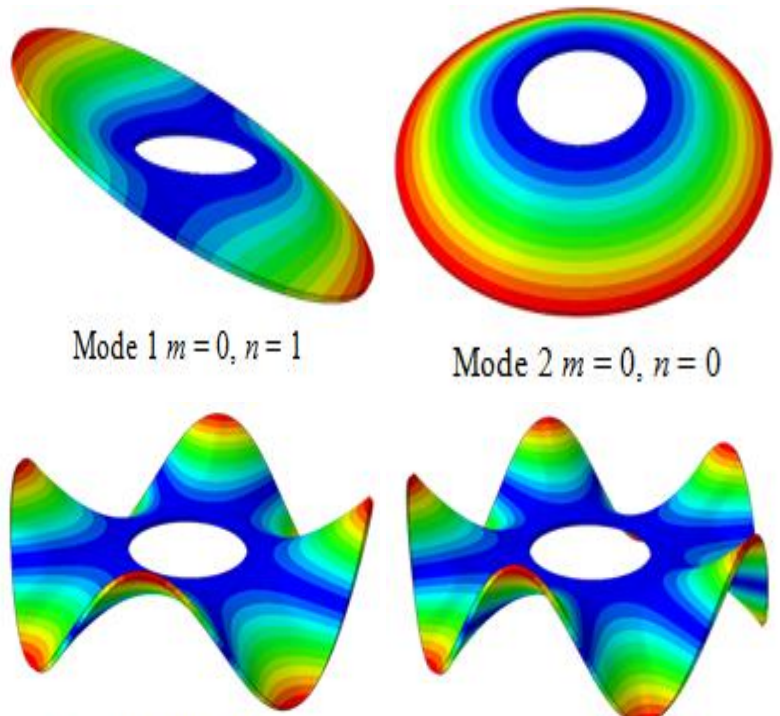

$$
\text { Mode } 2 m=0, n=0
$$

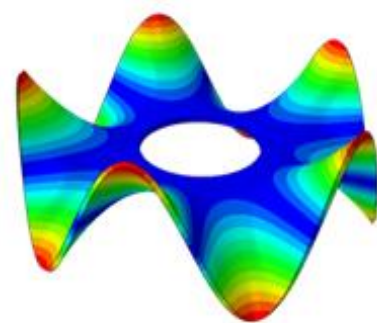

Mode $5 m=0, n=4$

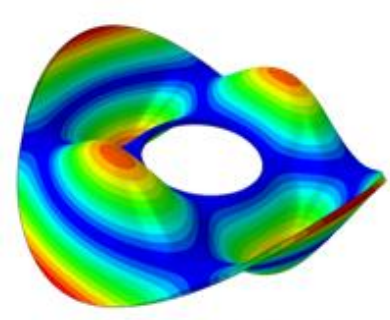

Mode $9 m=1, n=2$

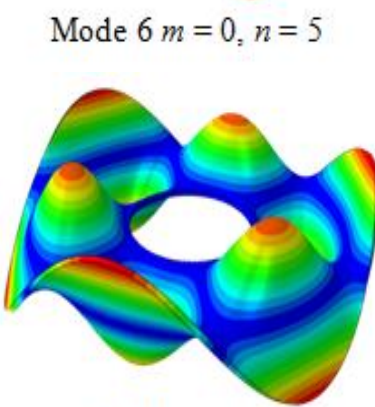

Mode $11 m=1, n=3$ changed if the rotational body force changes. Increased rotational speed will increase the natural frequencies.

Mesh convergence is done based on the discontinuities in the static step. Since the elements are quadratic quadriliteral elements a smooth and relatively continuous stress/displacement distribution is expected from the model.

The static step cannot include rotation itself but only the loads caused by centrifugal acceleration. Disk is loaded with centrifugal body forces and creates a similar stress field when disk actually rotates. So the stiffness matrix can be updated in order to include the effects of rotation into account and obtain proper results. Therefore the calculated frequencies require some additional operations to obtain the effects of travelling waves. $+\Omega^{*} n$ and $-\Omega^{*} n$ are to be added to the frequencies of the related vibration modes, where $\Omega$ is the rotation speed of the hard disk and $\mathrm{n}$ is the nodal diameter.



Mode $3 m=0, n=2$



Mode $4 m=0, n=3$
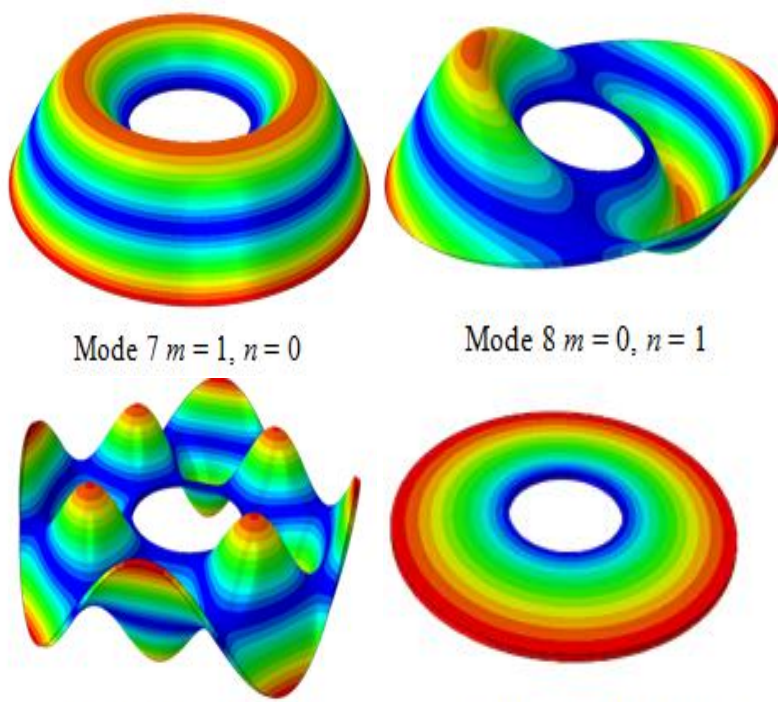

Mode $8 m=0, n=1$

Mode $13 m=1, n=4$
Mode 14 In-plane

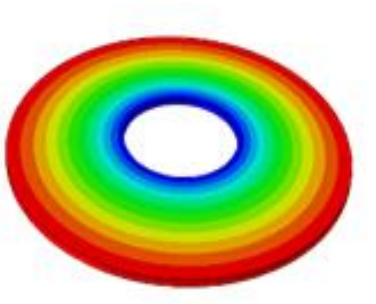

Fig. 6. Examples of mode shapes 
TABLE 2

NUMERICAL RESULTS FOR VARIOUS SPEEDS

\begin{tabular}{|c|c|c|c|c|c|c|c|}
\hline Rotation Speed (RPM) & Wave Type & $\begin{array}{c}\text { Mode } 1(\mathrm{~Hz}) \\
(0,1)\end{array}$ & $\begin{array}{c}\text { Mode } 2(\mathrm{~Hz}) \\
(0,0)\end{array}$ & $\begin{array}{c}\text { Mode } 3(\mathrm{~Hz}) \\
(0,2)\end{array}$ & $\begin{array}{c}\text { Mode } 4(\mathrm{~Hz}) \\
(0,3)\end{array}$ & $\begin{array}{c}\text { Mode } 5(\mathrm{~Hz}) \\
(0,4)\end{array}$ & $\begin{array}{c}\text { Mode } 6(\mathrm{~Hz}) \\
(0,5)\end{array}$ \\
\hline 0 & - & 1041,47 & 1050,37 & 1211,47 & 1878,12 & 3035,22 & 4576,47 \\
\hline 2000 & BTW & 1009,01 & 1051,11 & 1145,88 & 1779,18 & 2902,84 & 4410,66 \\
\hline \multirow[t]{2}{*}{4000} & FTW & 1111,62 & 1053,32 & 1349,14 & 2082,33 & 3305,67 & 4913,24 \\
\hline & BTW & 978,28 & 1053,32 & 1082,48 & 1682,33 & 2772,33 & 4246,58 \\
\hline 6000 & BTW & 949,30 & 1056,98 & 1021,21 & 1587,59 & 2643,71 & 4084,21 \\
\hline \multirow[t]{2}{*}{8000} & FTW & 1188,67 & 1062,10 & 1495,40 & 2294,92 & 3583,62 & 5256,89 \\
\hline & BTW & 922,01 & 1062,10 & 962,06 & 1494,92 & 2516,96 & 3923,55 \\
\hline
\end{tabular}

\section{DISCUSSION}

In order to be able to validate obtained experimental and numerical results, these resullts are compared to the analytical results from [13]. Exact values of frequencies of first six modes of a stationary and rotating disk are represented in Table 3. In the cases of rotating disk, forward and backward travelling waves at the given modes are included in these results. With increasing rotational speeds, forward and backward travelling waves become more distinct and their separation is to be observed more clearly. The presented analytical results are in good agreement with most studies in the literature $[8,10,12,14]$.

TABLE 3

ANALYTICAL RESULTS FOR VARIOUS SPEEDS

\begin{tabular}{lccccccc}
\hline \hline Rotation Speed (RPM) & Wave Type & $\begin{array}{c}\text { Mode 1 (Hz) } \\
(0,1)\end{array}$ & $\begin{array}{c}\text { Mode 2 (Hz) } \\
(0,0)\end{array}$ & $\begin{array}{c}\text { Mode 3 (Hz) } \\
(0,2)\end{array}$ & $\begin{array}{c}\text { Mode 4 (Hz) } \\
(0,3)\end{array}$ & $\begin{array}{c}\text { Mode 5 (Hz) } \\
(0,4)\end{array}$ & $\begin{array}{c}\text { Mode 6 (Hz) } \\
(0,5)\end{array}$ \\
\hline 0 & & 1044,769 & 1052,165 & 1218,798 & 1890,442 & 3056,188 & 4613,717 \\
2000 & - & 1078,974 & 1052,902 & 1286,546 & 1991,491 & 3190,46 & 4781,238 \\
& FTW & 1012,307 & 1052,902 & 1153,212 & 1791,491 & 2923,793 & 4447,905 \\
4000 & BTW & 1114,918 & 1055,11 & 1356,449 & 2094,632 & 3326,608 & 4950,468 \\
& FTW & 105,11 & 1089,783 & 1694,632 & 2793,274 & 4283,801 \\
6000 & BTW & 981,5843 & $1053,432,406$ \\
& FTW & 1152,587 & 1058,778 & 1428,492 & 2199,857 & 3464,626 & 5121,406 \\
8000 & BTW & 952,5872 & 1058,778 & 1028,492 & 1599,857 & 2664,626 & 4121,403 \\
& FTW & 1191,961 & 1063,892 & 1502,645 & 2307,147 & 3604,506 & 5294,039 \\
& BTW & 925,294 & 1063,892 & 969,3117 & 1507,147 & 2537,84 & 3960,705 \\
\hline \hline
\end{tabular}

Experimental and numerical results are compared to the presented analytical values in terms of absolute percentage error and presented in Table 4 and Table 5, respectively. It can be seen from the Table 4, that maximum error between experimental and analytical results is $12.841 \%$ in mode 1 at $8000 \mathrm{rpm}$. The least error is to be seen as $0.174 \%$ at the forward travelling wave of mode 3 at 8000 rpm rotational speed. The error values between experimental and analytical reults are greater for the first two modes. The reason for this lies in the experimental setup. Disk is rotated using a non rigid drving system including bearing, power transmission elements and electric motor. The fixtures where the HDD is located during the experimentation process, are also not rigid. The stiffness of drviving system and fixtures affect the first two modes of the disk so that a clean measurement is not easy to be made and the data could not be processed to remove the effects of the stiffness of other elements. After all it is still to be stated that the experimental and analytical results generally support each other. Also, Table 5 shows numerical and experimental results. The maximum error between these two sets of results is about $12.440 \%$ at backward travelling wave of mode 1 when rotating at $8000 \mathrm{rpm}$ and the least error is about $0.005 \%$ at forward travelling wave of mode 6 when rotating with $4000 \mathrm{rpm}$. The greater error margins for the first two modes are caused by the same reasons explained for the 
first two modes presented in Table 4. Therefore, agreement of numerical end experimental results is well to be noticed.

Last comparison, which is introduced in Table 6, is done between numerical and analytical results. Table 6 shows that maximum error between numerical and analyt- ical results is $0.938 \%$ in mode 6 at $8000 \mathrm{rpm}$. Minimum error between analytical and numerical results is $0.168 \%$ in mode 2 at $8000 \mathrm{rpm}$. These results show that numerical results are consistent with the analytical results.

TABLE 4

EXPERIMENTAL-ANALYTICAL RESULTS ABSOLUTE PERCENTAGE ERROR

\begin{tabular}{lccccccc}
\hline \hline Rotation Speed (RPM) & Wave Type & Mode 1 (Hz) & Mode 2 (Hz) & Mode 3 (Hz) & Mode 4 (Hz) & Mode 5 (Hz) & Mode 6 (Hz) \\
& & $(0,1)$ & $(0,0)$ & $(0,2)$ & $(0,3)$ & $(0,4)$ \\
\hline 0 & - & 9,400 & 7,380 & 1,072 & 3,303 & 1,568 \\
2000 & FTW & 10,891 & 7,721 & 1,111 & 3,669 & 1,445 \\
& BTW & 11,734 & 7,721 & 1,771 & 4,035 & 1,874 & 1,297 \\
4000 & FTW & 10,497 & 8,091 & 0,478 & 3,082 & 1,545 \\
& BTW & 11,544 & 8,091 & 1,019 & 3,268 & 1,907 & 1,388 \\
6000 & FTW & 10,932 & 8,172 & 0,315 & 2,941 & 1,453 \\
& BTW & 11,806 & 8,172 & 1,485 & 3,618 & 1,548 \\
8000 & FTW & 10,674 & 8,285 & 1,256 & 2,768 & 1,450 & 1,313 \\
& BTW & 12,841 & 8,285 & 0,174 & 3,655 & 1,457 & 1,514 \\
\hline \hline
\end{tabular}

TABLE 5

NUMERICAL-EXPERIMENTAL RESULTS ABSOLUTE PERCENTAGE ERROR

\begin{tabular}{lccccccc}
\hline \hline Rotation Speed (RPM) & Wave Type & Mode 1 (Hz) & Mode 2 (Hz) & Mode 3 (Hz) & Mode 4 (Hz) & Mode 5 (Hz) & Mode 6 (Hz) \\
& & $(0,1)$ & $(0,0)$ & $(0,2)$ & $(0,3)$ & $(0,4)$ & $(0,5)$ \\
\hline 0 & - & 0,316 & 0,171 & 0,601 & 0,652 & 0,686 & 0,807 \\
2000 & FTW & 0,306 & 0,170 & 0,570 & 0,618 & 0,657 & 0,779 \\
& BTW & 0,326 & 0,170 & 0,636 & 0,687 & 0,717 & 0,837 \\
4000 & FTW & 0,296 & 0,170 & 0,539 & 0,587 & 0,630 & 0,752 \\
& BTW & 0,336 & 0,170 & 0,670 & 0,726 & 0,750 & 0,869 \\
6000 & FTW & 0,285 & 0,170 & 0,510 & 0,558 & 0,604 & 0,726 \\
& BTW & 0,345 & 0,170 & 0,708 & 0,767 & 0,785 & 0,902 \\
& FTW & 0,276 & 0,168 & 0,482 & 0,530 & 0,579 & 0,702 \\
& BTW & 0,355 & 0,168 & 0,748 & 0,811 & 0,823 & 0,938 \\
\hline \hline
\end{tabular}

TABLE 6

EXPERIMENTAL-ANALYTICAL RESULTS ABSOLUTE PERCENTAGE ERROR

\begin{tabular}{lccccccc}
\hline \hline Rotation Speed (RPM) & Wave Type & Mode 1 (Hz) & Mode 2 (Hz) & Mode 3 (Hz) & Mode 4 (Hz) & Mode 5 (Hz) & Mode 6 (Hz) \\
& & $(0,1)$ & $(0,0)$ & $(0,2)$ & $(0,3)$ & $(0,4)$ & $(0,5)$ \\
\hline 0 & - & 1044,769 & 1052,165 & 1218,798 & 1890,442 & 3056,188 & 4613,717 \\
2000 & FTW & 1078,974 & 1052,902 & 1286,546 & 1991,491 & 3190,46 & 4781,238 \\
& BTW & 1012,307 & 1052,902 & 1153,212 & 1791,491 & 2923,793 & 4447,905 \\
4000 & FTW & 1114,918 & 1055,11 & 1356,449 & 2094,632 & 3326,608 & 4950,468 \\
& BTW & 981,5843 & 1055,11 & 1089,783 & 1694,632 & 2793,274 & 4283,801 \\
6000 & FTW & 1152,587 & 1058,778 & 1428,492 & 2199,857 & 3464,626 & 5121,406 \\
& BTW & 952,5872 & 1058,778 & 1028,492 & 1599,857 & 2664,626 & 4121,403 \\
8000 & FTW & 1191,961 & 1063,892 & 1502,645 & 2307,147 & 3604,506 & 5294,039 \\
& BTW & 925,294 & 1063,892 & 969,3117 & 1507,147 & 2537,84 & 3960,705 \\
\hline \hline
\end{tabular}


Also, Campbell diagrams which consist of experimental and analytical values are plotted in Figure 7 and Figure 8. Figure 7 is plotted to visualize the varying frequencies of first three modes with rotation. It is clear that analytical and experimental analyses are consistent with acceptable error margins. The error of the slopes of the curves is almost negligible. Main error is originated by some shifts between analytical and experimental curves. As it can be seen in these figures, analytical and experimental values are in good agreement.

Figure 8 has the same purpose with Figure 7 but it displays the same characteristics for the modes 4, 5 and 6. Errors between the curves have almost the same characteristic, the slopes are almost equal when comparing the curves for the same modes but there is a shift to be noticed. The shift here is more acceptable in this case.

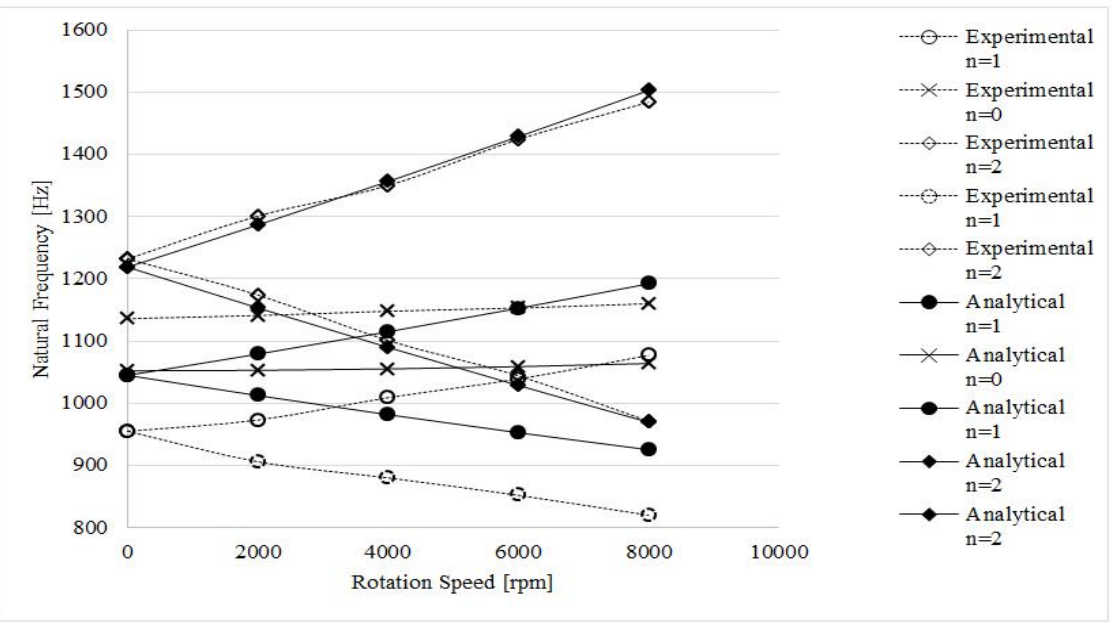

Fig. 7 . Campbell diagram of first 3 modes (Experimental-analytical)

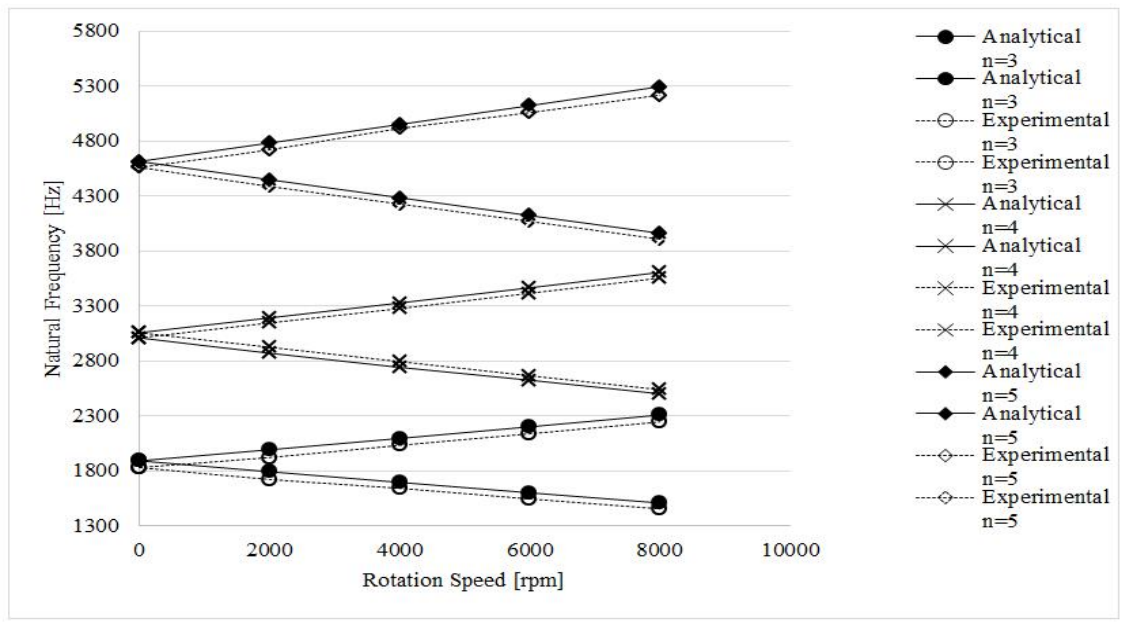

Fig. 8. Campbell diagram of $4^{\text {th }}, 5^{\text {th }}$ and $6^{\text {th }}$ modes (Experimental-analytical)

Going from the point that the numerical model is validated with analytical and experimental study, it is possible to manipulate the model for making further prediction about the mechanical characterization of the disk. With this idea, for getting an understanding of the behavior of the disk at higher rotation speed, the speed range is increased up to $30000 \mathrm{rpm}$ in numerical simulation.
These results are plotted in Figure 9, Figure 10 and Figure 11 to monitor the structural behavior at high speeds. Figure 9 displays the results for the modes at which $m=0$ and $n=0,1,2,3$. Figure 10 aims to illustrate the numerical results for the modes at which $m=0$ and $n=4,5,6$. Figure 11 shows numerical results for $m=1$ and $n=0,1,2$. 


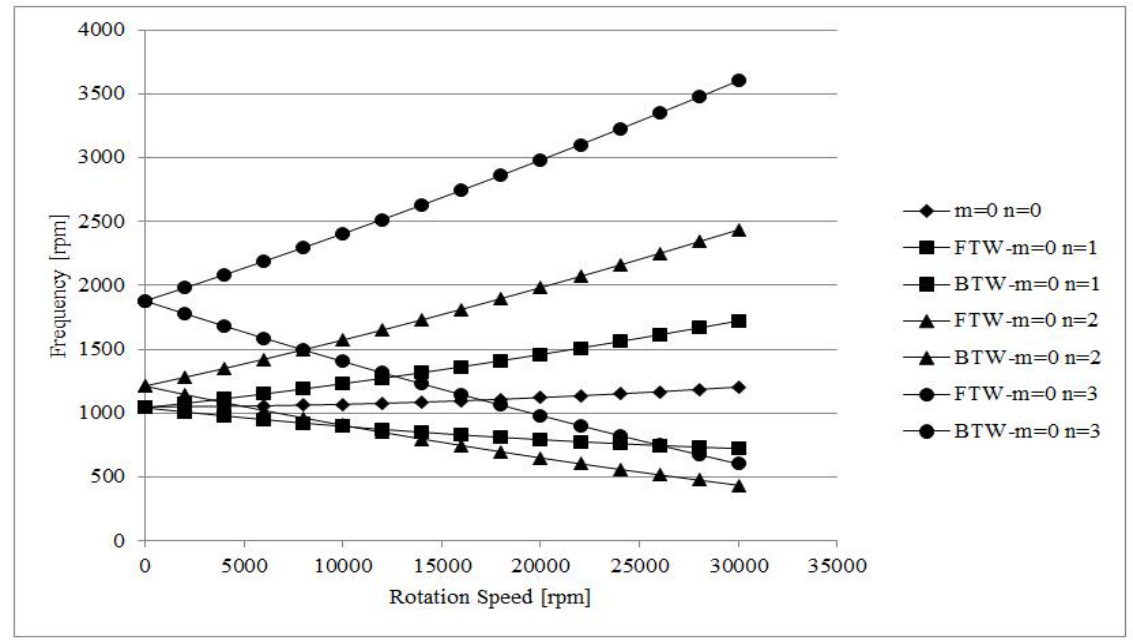

Fig. 9 . Campbell diagrams for numerically calculated modes $(0,0),(0,1),(0,2),(0,3)$

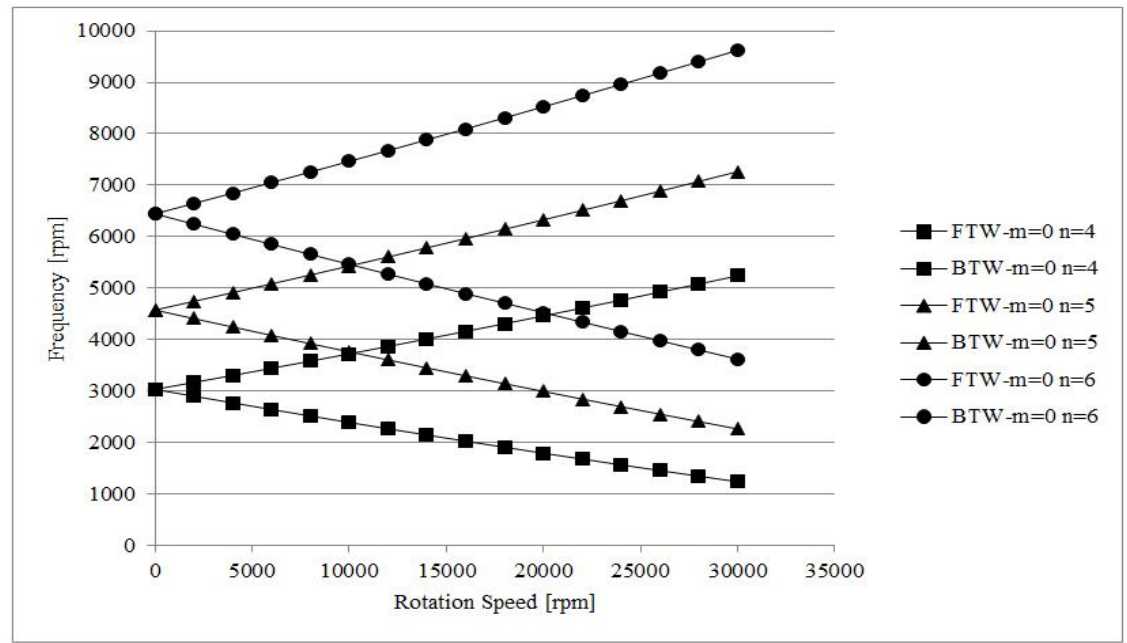

Fig. 10 . Campbell diagrams for numerically calculated modes $(0,4),(0,5),(0,6)$

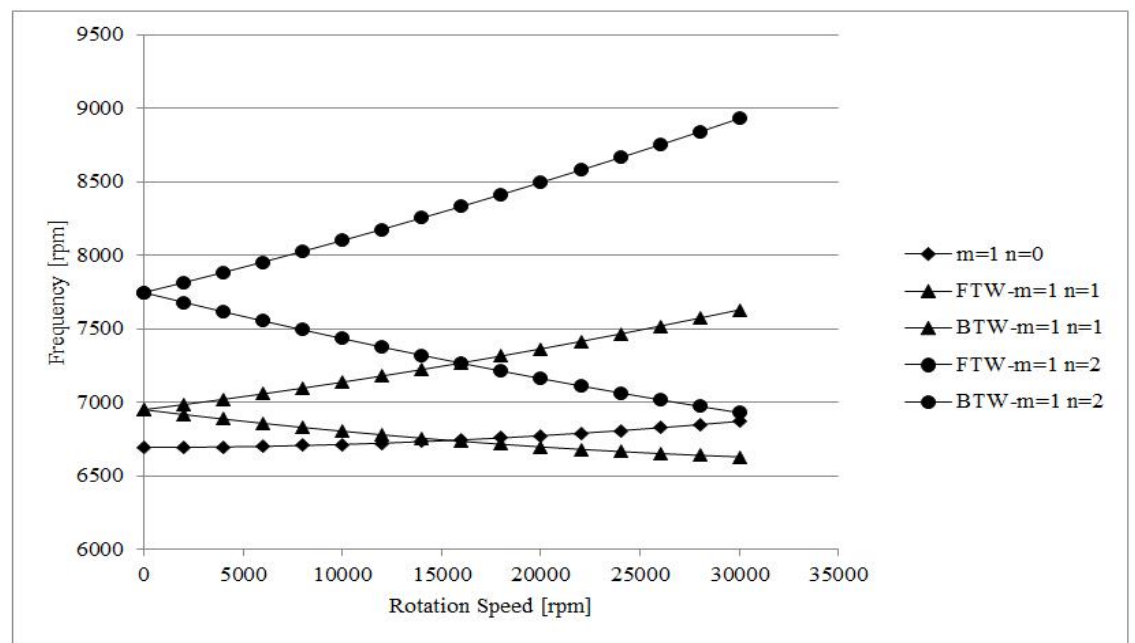

Fig. 11. Campbell diagrams for numerically calculated modes $(1,0),(1,1),(1,2)$ 


\section{CONCLUSION}

This study investigates the effects of rotation on the vibration characteristics of rotating disks. In order to get an understanding of the effects of rotation on disks, experimental and numerical studies are conducted. The experimental data are taken from a space fixed point so that the effects of travelling waves may be clearly seen. In the experimental data, there are some unpredicted frequencies that are the effects of driving systems, supports and casing of the hard disk drive. Additionally, numerical calculations are performed using finite elements approach. In this method the travelling waves are not to be observed since only the loads that are caused by rotation are taken into account, not rotation itself. Therefore, the travelling waves are obtained after manipulating the raw results.

In the experimental data, the first two frequencies are slightly different than those of numerical and analytical studies, while all other frequencies obtained analytically and numerically are in excellent agreement with those of experimental study. It is thought that with the stiffness of nonideal fixtures, driving systems lead to this difference. In analytical and numerical studies, the driving systems are not taken into account and the supports are idealized. Therefore the results of numerical approach are processed and the accurate results are obtained including travelling waves on the disk.

In general, the analytically and numerically predicted frequencies agree with the experimental results. The maximum error lies about $10 \%$.

\section{REFERENCES}

[1] H. Lamb and R. V. Southwell, "The vibrations of a spinning disk," in Proceedings of the Royal Society A, Mathematical, Physical and Engineering Sciences, vol. 99, pp. 272-280, 1921. DOI: 10.1098/rspa.1921.0041

[2] R. V. Southwell, "On the free transverse vibrations of a uniform circular disc clamped at its center, and on the effects of rotation," in Proceeding of the Royal Society A, Mathematical, Physical and Engineering Sciences, vol. 101, pp. 133-153, 1922. DOI: 10.1098/rspa.1922.0032

[3] J. L. Nowinski, "Nonlinear transverse vibrations of a spinning disk," Journal of Applied Mechanics, vol. 31, no. 1, pp. 72-78, 1964. DOI: /10.1115/1.3629573
[4] M. P. Mignolet, C. D. Eick and M. V. Harish, "Free vibration of flexible rotating disks," Journal of Sound and Vibration, vol. 196, no. 5, pp. 537-577, 1996.

DOI: $10.1006 /$ jsvi.1996.0502

[5] A. H. Nayfeh, A. Jilani and P. Manzione, "Transverse vibrations of a centrally clamped rotating circular disk," Nonlinear Dynamics, vol. 26, no. 2, pp. 163-178, 2001. DOI: $10.1023 / \mathrm{a}: 1012957024898$

[6] S. Bashmal, R. Bhat and S. Rakheja, "Experimental and numerical study of the vibration of stationary and rotating annular disks," Journal of Vibration and Acoustics, vol. 138, no. 5, pp. 051003, 2016.

DOI: $10.1115 / 1.4033359$

[7] I. Kajiwara and N. Hosoya, "Vibration measurement and monitoring of a rotating disk using contactless laser excitation," in Proceedings of the ASME 2013 Dynamic Systems and Control Conference, Stanford University, Munger Center, Palo Alto, CA, 2013.

[8] R. M. H. Khorasany and S. G. Hutton, "Vibration characteristics of rotating thin disks-part I: Experimental results," Journal of Applied Mechanics, vol. 79, no. 4, pp. 041006, 2012. DOI: 10.1115/1.4005539

[9] K. A. Cole and R. C. Benson, "A fast eigenfunction approach for computing spinning disk deflections," Journal of Applied Mechanics, vol. 55, no. 55, pp. 453-457, 1988. DOI: $10.1115 / 1.3173698$

[10] J. Kirkhope and G. J. Wilson, "Vibration and stress analysis of thin rotating discs using annular finite elements," Journal of Sound and Vibration, vol. 44, no. 4, pp. 461-474, 1976. DOI: 10.1016/0022-460x(76)90088-2

[11] L. M. Xu and N. Guo, "Modal testing and finite element modelling of subsystem in hard disk drive," Mechanical Systems and Signal Processing, vol. 17, no. 4, pp. 747-764, 2003. DOI: $10.1006 / \mathrm{mssp} .2002 .1508$

[12] C. S. Kim and C. W. Lee, "Travelling wave control of rotating discs and analysis of its spillover effect," Proceedings of the Institution of Mechanical Engineers, vol. 202, no. 2, pp. 119-127, 1988.

DOI: 10.1243/pime_proc_1988_202_097_02

[13] O. Oldac, M. Tufekci, O. E. Genel and E. Tufekci, "Transverse free vibrations of rotating annular plates," in Proceedings of Tokyo $18^{\text {th }}$ International Conference on Engineering \& Technology, Computer, Basic \& Applied Sciences, Tokyo, Japan, 2017. 
[14] W. D. Iwan and T. L. Moeller, "The stability of a spinning elastic disk with a transverse load system,"Journal of Ap- plied Mechanics, vol. 43, no. 3, pp. 485-490, 1976. DOI: $10.1115 / 1.3423896$

— This article does not have any appendix. — 